\title{
Cosmological Constant and Energy Density of Random Electromagnetic Field
}

\author{
Ilya A. Obukhov \\ Research \& Development Company "System Recourses", Moscow, Russia \\ Email: i_obukhov@systemres.ru \\ Received 14 April 2016; accepted 25 June 2016; published 28 June 2016 \\ Copyright (C) 2016 by author and Scientific Research Publishing Inc. \\ This work is licensed under the Creative Commons Attribution International License (CC BY). \\ http://creativecommons.org/licenses/by/4.0/ \\ (c) (i) 0pen Access
}

\begin{abstract}
It is shown that the non-equilibrium electrically neutral and relativistically invariant vacuum-like state with the negative energy density and positive pressure may exist at the non-zero temperature in the system of spinor particles, antiparticles, and random electromagnetic field generated by particle-particle, particle-antiparticle, and antiparticle-antiparticle transitions. At the temperature of the order of $10^{-5} \mathrm{~K}$, the energy density of its state corresponds to the dark energy density in absolute magnitude. The cosmological constant for such material medium turns out to be negative.
\end{abstract}

Keywords

Dark Energy, Cosmological Constant, Stochastic Electromagnetic Field, Spinor Particles

\section{Introduction}

Article [1] shows that the quantum theory allows building up the relativistically invariant state of matter, for which pressure $p$ and energy density $\varepsilon$ are related as follows

$$
p=-\varepsilon
$$

Interest in the investigation of such states is related to search for the physical interpretation of the cosmological constant in the Einstein's equations [1]-[3] and dark energy nature [4]-[6].

If a material medium exists, whose energy-momentum density tensor is expressible in the following form

$$
T_{\mu \nu}^{(\Lambda)}=g_{\mu \nu} \varepsilon^{(\Lambda)},
$$

then the cosmological constant $\Lambda$ in the Einstein's equations [1] can be related to the energy density of such medium $\varepsilon^{(\Lambda)}$ 


$$
\Lambda=\varepsilon^{(\Lambda)}\left(8 \pi G / c^{4}\right)
$$

Here $G$ is the gravitation constant, $c$ is the velocity of light. In case of the empty flat space, when the Minkovsky's tensor $\eta^{\mu v}$ with $(1,-1,-1,-1)$ diagonal is the metric tensor $g_{\mu v}$, energy density $\varepsilon^{(\Lambda)}$ and pressure $p^{(\Lambda)}$ obviously satisfy the relationship (1).

If $\varepsilon^{(\Lambda)}>0$, then negative pressure $p^{(\Lambda)}$ can be interpreted [4] [6] as the cause of the antigravity. The opposite possibility, where $\varepsilon^{(\Lambda)}<0$ [1] [2], is not also excluded. In such a case, the gravitational attraction between massive objects starts increasing at the distances exceeding some threshold one.

The cosmological constant problem is particularly relevant in the context of the experimental confirmation of the accelerated expansion of the Universe [6]. However, this phenomenon can be explained not only with the help of the General Relativity, but also by the different alternative versions of the Gravity Theory (see [7] and the articles cited there), where dark energy concept isn't introduced. In paper [7] it is shown that the analysis of the interference of gravitational waves will allow making a choice for that or another Gravity Theory.

Experimental discovery of gravitational waves [8] allows hoping for increasing of our understanding of the nature of gravitation. Data obtained now confirm justice of the General Relativity [8]. But it is only the first experiments and it is not enough for final conclusions.

In any case, up to date, the material medium model-building problem, for which relationship (1) is true and density

$$
\rho^{(\Lambda)}=\varepsilon^{(\Lambda)} / c^{2}
$$

is close, at least in the order-of-magnitude, to the value of

$$
\rho^{(\Lambda)} \approx(7.21 \pm 0.25) \times 10^{-30} \mathrm{~g} \cdot \mathrm{cm}^{-3}
$$

resulted from the interpretation of astrophysical data [6] that has not been solved. Solving this problem seems important for the understanding of potential physical phenomena leading to the formation of such material medium.

\section{Energy-Momentum Tensor of Random Electromagnetic Field}

Let us consider the model where the electromagnetic field tensor

$$
F^{\mu v}(x)=\partial^{\mu} A^{v}(x)-\partial^{v} A^{\mu}(x)
$$

is governed by the Maxwell's equations

$$
\partial_{\mu} F^{\mu v}(x)=(4 \pi e / c) q^{v}(x),
$$

with random source $q^{v}(x)$ on the right side. Here $A^{\mu}(x)$ is the vector potential of electromagnetic field, and $e$ is the electric charge.

We consider that for flow density $q^{\mu}(x)$ the conservation law is true

$$
\partial_{\mu} q^{\mu}(x)=0,
$$

and for potential $A^{\mu}(x)$ the Lorentz' gage condition is true

$$
\partial_{\mu} A^{\mu}(x)=0 .
$$

Suppose, devices available enable measuring only some time interval and/or volume mean values, which will be indicated with brackets $\langle\ldots .$.$\rangle . Suppose that$

$$
\left\langle q^{\mu}(x)\right\rangle=0,
$$

but at the same time for decomposition components $q^{\mu}(x)$ on some set of functions $q^{\mu}\left(x, \lambda, \lambda^{\prime}\right)$,

$$
q^{\mu}(x)=\sum_{\lambda} \sum_{\lambda^{\prime}} q^{\mu}\left(x, \lambda, \lambda^{\prime}\right)
$$

the following relationship is true:

$$
\begin{aligned}
& \left\langle\int \mathrm{d}^{4} x^{\prime \prime} \int \mathrm{d}^{4} x^{\prime} q^{* \mu}\left(x^{\prime \prime}, \sigma, \sigma^{\prime}\right) q^{v}\left(x^{\prime}, \lambda, \lambda^{\prime}\right) \exp \left\{-i k_{\alpha} x^{\prime \alpha}+i \chi_{\alpha} x^{\prime \prime}\right\}\right\rangle, \\
& =\delta_{\lambda \sigma} \delta_{\lambda^{\prime} \sigma^{\prime}} n^{\mu \nu}\left(k, \sigma, \sigma^{\prime} ; \chi, \lambda, \lambda^{\prime}\right)
\end{aligned}
$$


where $n^{\mu v}\left(k, \sigma, \sigma^{\prime} ; \chi, \lambda, \lambda^{\prime}\right)$ is the tensor, whose exact form will be defined later on.

The formal solution of Equations (6) for $A^{\mu}(x)$ meeting the initial condition

$$
A^{\mu}(x)=0 \text { at } t<0,
$$

taking into account Decomposition (10), can be written down in the following form

$$
A^{\mu}(x)=-(4 \pi e / c)(2 \pi)^{-4} \sum_{\lambda} \sum_{\lambda^{\prime}} \int \mathrm{d}^{4} \chi \int \mathrm{d}^{4} x^{\prime} q^{\mu}\left(x, \lambda, \lambda^{\prime}\right) \exp \left\{-i \chi_{v}\left(x^{v}-x^{\prime \nu}\right)\right\} /\left(\chi^{k} \chi_{k}+2 i \alpha \chi_{0}\right),
$$

where $\alpha>0, \alpha \rightarrow 0$. According to Equations (5), (9), and (13)

$$
\left\langle A^{\mu}(x)\right\rangle=0,\left\langle F^{\mu v}(x)\right\rangle=0 \text {. }
$$

Let us determine energy-momentum density tensors of electromagnetic field $F^{\mu v}(x)$ and its interaction with charged flow $q^{v}(x)$

$$
\begin{gathered}
t^{\mu v}(A(x))=-(4 \pi c)^{-1}\left(F^{\mu k}(x) F^{v}{ }_{k}(x)-(1 / 4) \eta^{\mu v} F^{k l}(x) F_{k l}(x)\right), \\
t^{\mu v}(q(x), A(x))=-\left(e / c^{2}\right) q^{\mu}(x) A^{v}(x) .
\end{gathered}
$$

These tensors can be presented using quadratic combinations $q^{v}(x)$, and therefore their average values may be non-zero. Using Formula (11), for the average value of the sum of tensors $t^{\mu v}(A(x))$ and $t^{\mu v}(q(x), A(x))$, we obtain

$$
\begin{aligned}
T^{(s) \mu v}(x) & =\left\langle t^{\mu v}(A(x))\right\rangle+\left\langle t^{\mu v}(q(x), A(x))\right\rangle \\
& =\sum_{\lambda} \sum_{\lambda^{\prime}} \int \mathrm{d}^{4} \chi \int \mathrm{d}^{4} k T^{(s) \mu v}\left(k, \chi ; \lambda, \lambda^{\prime}\right) \exp \left\{-i\left(\chi_{l}-k_{l}\right) x^{l}\right\}, \\
T^{(s) \mu v}\left(k, \chi ; \lambda, \lambda^{\prime}\right)=- & \left(4 \pi e^{2} / c^{3}\right)(2 \pi)^{-8}\left(\left(k^{\alpha} k_{\alpha}-2 i \alpha k_{0}\right)\left(\chi^{\alpha} \chi_{\alpha}+2 i \alpha \chi_{0}\right)\right)^{-1} \\
& \times\left\{\left(k^{\mu} \chi^{v}-(1 / 2) \eta^{\mu v} k^{\beta} \chi_{\beta}\right) n_{\alpha}^{\alpha}\left(k, \lambda, \lambda^{\prime} ; \chi, \lambda, \lambda^{\prime}\right)\right. \\
& +\left(k^{\alpha} \chi_{\alpha}-\left(k^{\alpha} k_{\alpha}-2 i \alpha k_{0}\right)\right) n^{\mu v}\left(k, \lambda, \lambda^{\prime} ; \chi, \lambda, \lambda^{\prime}\right)-k^{\alpha} \chi^{v} n_{\alpha}^{\mu}\left(k, \lambda, \lambda^{\prime} ; \chi, \lambda, \lambda^{\prime}\right) \\
& \left.-k^{\mu} \chi_{\alpha} n^{\alpha v}\left(k, \lambda, \lambda^{\prime} ; \chi, \lambda, \lambda^{\prime}\right)+(1 / 2) \eta^{\mu v} k^{\alpha} \chi_{\beta} n^{\beta}{ }_{\alpha}\left(k, \lambda, \lambda^{\prime} ; \chi, \lambda, \lambda^{\prime}\right)\right\} .
\end{aligned}
$$

Suppose, tensor $n^{\mu v}\left(k, \lambda, \lambda^{\prime} ; \chi, \lambda, \lambda^{\prime}\right)$ describes transitions between acceptable states of spinor particles and antiparticles with mass $m$ and charges $e$ and $-e$ in the binary mixture of their gases. Then it can be defined using the following relationships

$$
\begin{aligned}
& n^{\mu v}\left(k, \lambda, \lambda^{\prime} ; \chi, \lambda, \lambda^{\prime}\right)=c^{2}(m c / 2 \pi \hbar)^{6}(2 \pi)^{8}\left[n_{\left(r, r^{\prime}\right)}^{(e, e)}{ }^{\mu v}\left(k, \chi ; p, p^{\prime}\right)\right. \\
& \left.+n_{\left(r, r^{\prime}\right)}^{(h, h v}\left(k, \chi ; p, p^{\prime}\right)+n_{\left(r, r^{\prime}\right)}^{(h, e)}\left(k, \chi ; p, p^{\prime}\right)\right], \\
& n_{\left(r, r^{\prime}\right)}{ }^{\mu \nu}\left(k, \chi ; p, p^{\prime}\right)=Q^{(e, e)}\left(p, p^{\prime}, F^{(e)}, \Theta\right) \delta^{4}\left(k-\left(p-p^{\prime}\right) / \hbar\right) \delta^{4}\left(\chi-\left(p-p^{\prime}\right) / \hbar\right) \\
& \times w_{\left(r, r^{\prime}\right)}^{*(e e)}\left(p, p^{\prime}\right) w_{\left(r, r^{\prime}\right)}^{(e, e)}\left(p, p^{\prime}\right), \\
& n_{\left(r, r^{\prime}\right)}{ }^{\mu v}\left(k, \chi ; p, p^{\prime}\right)=Q^{(h, h)}\left(p, p^{\prime}, F^{(h)}, \Theta\right) \delta^{4}\left(k-\left(p-p^{\prime}\right) / \hbar\right) \delta^{4}\left(\chi-\left(p-p^{\prime}\right) / \hbar\right) \\
& \times w_{\left(r, r^{\prime}\right)}{ }^{*(h, h)}\left(p, p^{\prime}\right) w_{\left(r, r^{\prime}\right)}^{(h, h)}{ }^{v}\left(p, p^{\prime}\right), \\
& n_{\left(r, r^{\prime}\right)}^{(h, e)}\left(k, \chi ; p, p^{\prime}\right)=Q^{(h, e)}\left(p, p^{\prime}, F^{(e)}, F^{(h)}, \Theta\right) \delta^{4}\left(k-\left(p-p^{\prime}\right) / \hbar\right) \delta^{4}\left(\chi-\left(p-p^{\prime}\right) / \hbar\right) \\
& \times w_{\left(r, r^{\prime}\right)}^{*(h, e)}\left(p, p^{\prime}\right) w_{\left(r, r^{\prime}\right)}^{(h, e)}{ }^{v}\left(p, p^{\prime}\right), \\
& Q^{(e, e)}\left(p, p^{\prime}, F^{(e)}, \Theta\right)=\theta\left(\left(E^{(e)}\left(p^{\prime}\right)-E^{(e)}(p)\right) / m c^{2}\right) f\left(s_{e}\left(p^{\prime}, F^{(e)}, \Theta\right)\right)\left(1-f\left(s_{e}\left(p, F^{(e)}, \Theta\right)\right)\right), \\
& Q^{(h, h)}\left(p, p^{\prime}, F^{(h)}, \Theta\right)=\theta\left(\left(E^{(h)}(p)-E^{(h)}\left(p^{\prime}\right)\right) / m c^{2}\right) f\left(s_{h}\left(p, F^{(h)}, \Theta\right)\right)\left(1-f\left(s_{h}\left(p^{\prime}, F^{(h)}, \Theta\right)\right)\right),
\end{aligned}
$$




$$
Q^{(h, e)}\left(p, p^{\prime}, F^{(e)}, F^{(h)}, \Theta\right)=f\left(s_{e}\left(p^{\prime}, F^{(e)}, \Theta\right)\right) f\left(s_{h}\left(p, F^{(h)}, \Theta\right)\right) .
$$

Here indices $e$ and $h$ pertain to particles and antiparticles, respectively; $\hbar$ is the Plank's constant divided by $2 \pi ; p^{\mu}$, and $p^{\prime \mu}$ are four-dimensional momenta of particles and antiparticles, for which the following dispersion relationship is true

$$
p_{0}^{2}=\left(m c^{2}+p^{2}\right)^{1 / 2}>0, p^{2}=(\boldsymbol{p}, \boldsymbol{p}),
$$

indices $r$ and $r^{\prime}$ take on the values of \pm 1 and correspond to the two possible spin states of particles and antiparticles; $F^{(e)}$ and $F^{(h)}$ are chemical potentials of particles and antiparticles, which we will consider as constant;

$$
\Theta=k_{B} T,
$$

$k_{B}$ is the Boltzmann's constant, $T$ is the temperature;

$$
f(s)=(\exp \{s\}+1)^{-1}
$$

the Fermi-Dirac distribution function;

$$
s_{e}=\left(p_{\mu} U^{\mu}-F^{(e)}\right) / \Theta, s_{h}=\left(p_{\mu} U^{\mu}+F^{(h)}\right) / \Theta,
$$

$U^{\mu}$ is the hydrodynamic velocity [9] that meets the relationship

$$
U_{\mu} U^{\mu}=c^{2}, U_{0}>0 \text {. }
$$

Vectors $w_{\left(r, r^{\prime}\right)}^{(a, b)}\left(p, p^{\prime}\right)$ correspond to flows of transitions between the states of particles and antiparticles

$$
\begin{aligned}
& w_{(r, s)}^{(e, e)}{ }^{\mu}\left(p, p^{\prime}\right)=\bar{u}_{r}^{(e)}(p) \gamma^{\mu} u^{(e)}\left(p^{\prime}\right), w_{(r, s)}^{(h, h)}{ }^{\mu}\left(p, p^{\prime}\right)=\bar{u}_{r}^{(h)}(p) \gamma^{\mu} u^{(h)}{ }_{s}\left(p^{\prime}\right), \\
& w_{(h, s)}^{(h, e)}\left(p, p^{\prime}\right)=\bar{u}_{r}^{(h)}(p) \gamma^{\mu} u_{s}^{(e)}{ }_{s}\left(p^{\prime}\right),
\end{aligned}
$$

where $\gamma^{\mu}$ is the Dirac gamma-matrices, $u_{r}^{(e)}(p)$ and $u_{r}^{(h)}(p)$ are spinors that satisfy Equations

$$
\left(\gamma^{\mu} p_{\mu}-m c\right) u_{r}^{(e)}(p)=0,\left(\gamma^{\mu} p_{\mu}+m c\right) u_{r}^{(h)}(p)=0,
$$

and normalization conditions

$$
\bar{u}_{r}^{(e)}(p) u^{(e)}{ }_{s}(p)=\delta_{r s}, \bar{u}_{r}^{(h)}(p) u^{(h)}{ }_{s}(p)=-\delta_{r s}
$$

It is easy to show that the relations of orthogonality are true

$$
\left(p_{\mu}^{\prime}-p_{\mu}\right) w_{(r, s)}^{(e, e)}{ }^{\mu}\left(p, p^{\prime}\right)=0,\left(p_{\mu}^{\prime}-p_{\mu}\right) w_{(r, s)}^{(h, h)}{ }^{\mu}\left(p, p^{\prime}\right)=0, \quad\left(p_{\mu}^{\prime}+p_{\mu}\right) w_{(r, s)}^{(h, e)}{ }^{\mu}\left(p, p^{\prime}\right)=0 .
$$

With account of these definitions, Expressions (25), and the formula for the transition from the summation over composite index $\lambda$ to the integration over momentum $p$

$$
\sum_{\lambda}(\ldots) \rightarrow(m c)^{-2} \sum_{r} \int \mathrm{d}^{4} p \theta\left(p_{0} / m c\right) \delta\left(p_{\mu} p^{\mu}-m^{2} c^{2}\right)(\ldots)=(m c)^{-2} \sum_{r} \int\left(\mathrm{d}^{3} p / p_{0}\right)(\ldots),
$$

we obtain from Relationships (16.1) and (16.2)

$$
\begin{gathered}
T^{(s) \mu v}(x)=T^{(e, e) \mu v}(x)+T^{(h, h) \mu v}(x)+T^{(h, e) \mu v}(x), \\
T^{(a, b) \mu v}(x)=-\left(\alpha / 2 \pi^{2}\right)(2 \pi \hbar)^{-3}(m c)^{2} \sum_{r} \sum_{r^{\prime}} \int\left(\mathrm{d}^{3} p^{\prime} / p_{0}^{\prime}\right) \int\left(\mathrm{d}^{3} p / p_{0}\right) T^{(a, b) \mu v}\left(p^{\prime}, r^{\prime}, p, r\right), a, b=e, h, \\
T^{(e, e) \mu v}\left(p^{\prime}, r^{\prime}, p, r\right) \\
=\theta\left(\left(E^{(e)}\left(p^{\prime}\right)-E^{(e)}(p)\right) / m c^{2}\right) f\left(s_{e}\left(p^{\prime}, F^{(e)}, \Theta\right)\right)\left(1-f\left(s_{e}\left(p, F^{(e)}, \Theta\right)\right)\right) \\
\times w_{\left(r, r^{\prime}\right)}^{\beta}\left(p, p^{\prime}\right) w_{\left(r, r^{\prime}\right) \beta}^{(e, e)}\left(p, p^{\prime}\right)\left(n^{\mu} n^{v}-(1 / 2) \eta^{\mu v} n^{\beta} n_{\beta}\right)\left(n^{\beta} n_{\beta}\right)^{-2}, n^{\mu}=p^{\prime \mu}-p^{\mu},
\end{gathered}
$$




$$
\begin{aligned}
& T^{(h, h) \mu v}\left(p^{\prime}, r^{\prime}, p, r\right) \\
& =\theta\left(\left(E^{(h)}(p)-E^{(h)}\left(p^{\prime}\right)\right) / m c^{2}\right) f\left(s_{h}\left(p, F^{(h)}, \Theta\right)\right)\left(1-f\left(s_{h}\left(p^{\prime}, F^{(h)}, \Theta\right)\right)\right) \\
& \times w_{\left(r, r^{\prime}\right)}^{*(h, h)}\left(p, p^{\prime}\right) w_{\left(r, r^{\prime}\right) \beta}^{(h, h)}\left(p, p^{\prime}\right)\left(n^{\mu} n^{v}-(1 / 2) \eta^{\mu v} n^{\beta} n_{\beta}\right)\left(n^{\beta} n_{\beta}\right)^{-2}, n^{\mu}=p^{\mu}-p^{\prime \mu}, \\
& T^{(h, e) \mu v}\left(p^{\prime}, r^{\prime}, p, r\right)=f\left(s_{e}\left(p^{\prime}, F^{(e)}, \Theta\right)\right) f\left(s_{h}\left(p, F^{(h)}, \Theta\right)\right) w_{\left(r, r^{\prime}\right)}^{*(h, e)}\left(p, p^{\prime}\right) \\
& \times w_{\left(r, r^{\prime}\right) \beta}^{(h, e)}\left(p, p^{\prime}\right)\left(n^{\mu} n^{v}-(1 / 2) \eta^{\mu v} n^{\beta} n_{\beta}\right)\left(n^{\beta} n_{\beta}\right)^{-2}, n^{\mu}=p^{\mu}+p^{\prime \mu},
\end{aligned}
$$

where $\alpha=e^{2} / \hbar c$ is the fine-structure constant approximately equal to $1 / 137$, if $e$ is the electron charge.

\section{Energy Density and Pressure of Interacting Mixture}

Assume, the inequations are true

$$
F^{(e)}>m c^{2}, F^{(h)}<-m c^{2},
$$

i.e. particle and antiparticle gases are degenerating ones. Let us consider the range of temperature and chemical potential values that satisfy the following conditions:

$$
p_{F}^{(e)} 2 / m \ll \Theta \ll m c^{2}, p_{F}^{(h)}{ }^{2} / m \ll \Theta \ll m c^{2} .
$$

Here $p_{F}^{(e)}$ and $p_{F}^{(h)}$ are Fermi momenta defined in the following relationships:

$$
p_{F}^{(e)}=c^{-1}\left(F^{(e) 2}-m^{2} c^{4}\right)^{1 / 2}, p_{F}^{(h)}=c^{-1}\left(F^{(h) 2}-m^{2} c^{4}\right)^{1 / 2} .
$$

In the frame of reference where $\boldsymbol{U}=0$, we obtain the expressions for energy density and pressure

$$
\begin{gathered}
\varepsilon^{(s)} \approx-(8 \pi)(4 / \pi-1) \alpha c(2 \pi \hbar)^{-3}(m \Theta)^{2}=-\pi^{-2}(4 / \pi-1)\left(e m k_{B} T / \hbar^{2}\right)^{2}, \\
p^{(s)} \approx-\varepsilon^{(s)} / 3 \approx(8 \pi / 3)(4 / \pi-1) \alpha c(2 \pi \hbar)^{-3}(m \Theta)^{2}=\left(3 \pi^{2}\right)^{-1}(4 / \pi-1)\left(e m k_{B} T / \hbar^{2}\right)^{2}
\end{gathered}
$$

related to the components of the energy-momentum tensor by simple relationships

$$
\varepsilon^{(s)}=c T^{(s) 00}, p^{(s)}=-(c / 3)\left(T_{\mu}^{(s) \mu}-T_{0}^{(s) 0}\right) .
$$

The following formulas are true under Conditions (28) in the same frame of reference for concentrations of particles and antiparticles $n^{(e)}$ and $n^{(h)}$, their energy densities and pressures

$$
\begin{gathered}
n^{(e)} \approx(8 \pi / 3)\left(p_{F}^{(e)} / 2 \pi \hbar\right)^{3}, n^{(h)} \approx(8 \pi / 3)\left(p_{F}^{(h)} / 2 \pi \hbar\right)^{3}, \\
\varepsilon^{(e)} \approx(8 \pi / 3) m c^{2}\left(p_{F}^{(e)} / 2 \pi \hbar\right)^{3}=m c^{2} n^{(e)}, \varepsilon^{(h)} \approx(8 \pi / 3) m c^{2}\left(p_{F}^{(h)} / 2 \pi \hbar\right)^{3}=m c^{2} n^{(h)}, \\
p^{(e)} \approx(16 \pi / 15)\left(p_{F}^{(e) 2} / 2 m\right)\left(p_{F}^{(e)} / 2 \pi \hbar\right)^{3}=(2 / 5)\left(p_{F}^{(e)}{ }_{F}^{2} / 2 m\right) n^{(e)}, \\
p^{(h)} \approx(16 \pi / 15)\left(p_{F}^{(h)}{ }^{2} / 2 m\right)\left(p_{F}^{(h)} / 2 \pi \hbar\right)^{3}=(2 / 5)\left(p_{F}^{(h)} 2 / 2 m\right) n^{(h)} .
\end{gathered}
$$

The condition of electroneutrality of the particle and antiparticle gases mixture has the following form

$$
F^{(e)}+F^{(h)}=0,
$$

whence it follows that

$$
p_{F}^{(e)}=p_{F}^{(h)}=p_{F} .
$$

In such a case, we obtain for the total energy density and pressure

$$
\varepsilon=\varepsilon^{(e)}+\varepsilon^{(h)}+\varepsilon^{(s)} \approx(16 \pi / 3) m c^{2}\left(p_{F}^{(e)} / 2 \pi \hbar\right)^{3}-(8 \pi)(4 / \pi-1) \alpha c(2 \pi \hbar)^{-3}(m \Theta)^{2},
$$




$$
p=p^{(e)}+p^{(h)}+p^{(s)} \approx(8 \pi / 3)(4 / \pi-1) \alpha c(2 \pi \hbar)^{-3}(m \Theta)^{2} .
$$

The energy density and pressure of electrically neutral particle and antiparticle gases mixture will be relativistically invariant in two cases [1] where

$$
\varepsilon=0, p=0
$$

and where condition (1) is met. In the first case, we obtain from Equations (35.1) and (35.2)

$$
T=0, p_{F}=0 .
$$

Furthermore, according to Formulas (32.1), concentrations of particles and antiparticles are zero.

In the second case, we obtain the condition of implementing the electrically neutral and relativistically invariant state for the mixture under consideration from Relationships (1), (35.1) and (35.2)

$$
p_{\Lambda}{ }^{3}=(4 / \pi-1) \alpha(m c)^{3}\left(\Theta_{\Lambda} / m c^{2}\right)^{2}=(4 / \pi-1)\left(e^{2}{k_{B}}^{2} T_{\Lambda}{ }^{2} m / \hbar c^{2}\right) .
$$

Here the Fermi momentum and temperature, at which conditions (1) is met, are indicated using $p_{\Lambda}$ and $T_{\Lambda}$. It is easy to show that momentum $p_{\Lambda}$ satisfying Equation (37) satisfies also Inequations (28)

$$
p_{\Lambda}{ }^{2} \ll m \Theta_{\Lambda} \Rightarrow \alpha(m c)^{3}\left(\Theta_{\Lambda} / m c^{2}\right)^{2} \ll\left(m \Theta_{\Lambda}\right)^{3 / 2} \Rightarrow \alpha\left(\Theta_{\Lambda} / m c^{2}\right)^{1 / 2} \ll 1
$$

The following expressions are true for the concentrations of particles and antiparticles, energy density and pressure of the mixture

$$
\begin{gathered}
n^{(e)}=n^{(h)}=n^{(\Lambda)} \approx(8 \pi / 3)\left(p_{\Lambda} / 2 \pi \hbar\right)^{3}=\left(3 \pi^{2}\right)^{-1}(4 / \pi-1) \alpha\left(\Theta_{\Lambda}{ }^{2} m / c \hbar^{3}\right) \\
=\left(3 \pi^{2}\right)^{-1}(4 / \pi-1) m\left(e k_{B} T_{\Lambda} / \hbar^{2} c\right)^{2}, \\
\varepsilon^{(\Lambda)} \approx-p^{(\Lambda)} \approx-(8 \pi / 3) \alpha c(2 \pi \hbar)^{-3}(4 / \pi-1)\left(m \Theta_{\Lambda}\right)^{2} \\
=-\left(3 \pi^{2}\right)^{-1}(4 / \pi-1)\left(e m k_{B} T_{\Lambda} / \hbar^{2}\right)^{2}=-m c^{2} n^{(\Lambda)} .
\end{gathered}
$$

We receive the following from Formulas (3), (4), and (38.2) for the cosmological constant and dark energy density

$$
\begin{gathered}
\Lambda \approx-\left(8 \pi G m / c^{2}\right) n^{(\Lambda)}=-(8 / 3 \pi)(4 / \pi-1)\left(G m^{2} e^{2}{k_{B}}^{2} T_{\Lambda}{ }^{2}\right) /(\hbar c)^{4} \\
=-(8 / 3 \pi)(4 / \pi-1) \alpha \alpha_{G}\left(k_{B} T_{\Lambda} / \hbar c\right)^{2}=-(8 / 3 \pi)(4 / \pi-1) \alpha \alpha_{G} R_{T}^{-2}, \\
\rho^{(\Lambda)}=-(8 \pi / 3)(4 / \pi-1)(\alpha / c)(2 \pi \hbar)^{-3}\left(m \Theta_{\Lambda}\right)^{2}=-\left(3 \pi^{2}\right)^{-1}(4 / \pi-1) \alpha m R_{e}^{-1} R_{T}{ }^{-2} \\
=-\left(3 \pi^{2}\right)^{-1}(4 / \pi-1)\left(e m k_{B} T_{\Lambda} / \hbar^{2} c\right)^{2}=-m n^{(\Lambda)} .
\end{gathered}
$$

Here the following is specified

$$
\alpha_{G}=G m^{2} / \hbar c,
$$

- $a$ dimensionless value representing $a$ gravitational analogue of the fine structure constant in terms of the build-up method, if $m$ is the mass of electron, then $\alpha_{G} \approx 1.75 \times 10^{-45}$;

$$
R_{T}=\hbar c / k_{B} T_{\Lambda}
$$

is the analogue of the Compton wave-length

$$
R_{e}=\hbar / m c
$$

for an object with the energy equal to $\Theta_{\Lambda}$.

If $e$ and $m$ are the charge and mass of electron, and dark energy density

$$
\begin{gathered}
\rho^{(\Lambda)}=-7.21 \times 10^{-30} \mathrm{~g} \cdot \mathrm{cm}^{-3}, \text { then } \Lambda \approx 1.34 \times 10^{-56} \mathrm{~cm}^{-2}, T_{\Lambda} \approx 1.54 \times 10^{-5} \mathrm{~K}, \\
R_{T} \approx 1.49 \times 10^{4} \mathrm{~cm}=149 \mathrm{~m}, p_{\Lambda} \approx 6.5 \times 10^{-28} \mathrm{~g} \cdot \mathrm{cm} / \mathrm{s}, v_{\Lambda}=p_{\Lambda} / \mathrm{m} \approx 0.71 \mathrm{~cm} / \mathrm{s} .
\end{gathered}
$$


The second of Formulas (39.2) for density $\rho^{(\Lambda)}$ has the strongly non-isotropic form. As if the dark energy is distributed in a parallelepiped with the very small height $R_{e}$ as compared with the typical dimensions of edges of bases $R_{T}$

$$
R_{e} / R_{T} \approx 2.5 \times 10^{-15} .
$$

Note that the contribution of the random electromagnetic field, including the contribution of interaction of this field with particles and antiparticles, to the total energy density in absolute magnitude is thrice as much as the contributions of particles and antiparticles equal to each other

$$
\varepsilon^{(e)}=\varepsilon^{(h)}=m c^{2} n^{(\Lambda)}, \varepsilon^{(s)}=-3 m c^{2} n^{(\Lambda)} .
$$

Just due to the random electromagnetic field generated by transitions between particles and antiparticles, the state that satisfies Condition (1) is possible in the system studied.

In the approximation considered, energy density $\varepsilon^{(s)}$ and pressure $p^{(s)}$ are resulting mainly from particleparticle and antiparticle-antiparticle transitions. The energy density and pressure related to particle-antiparticle transitions are much less than $\varepsilon^{(e, e)}, \varepsilon^{(h, h)}$, and $p^{(e, e)}, p^{(h, h)}$

$$
\varepsilon^{(h, e)} \approx p^{(h, e)} \approx-(32 / 9) \alpha c\left(p_{F}{ }^{3} / m^{2} c^{2}\right)\left(p_{F} / 2 \pi \hbar\right)^{3},\left|\varepsilon^{(h, e)} / \varepsilon^{(s)}\right| \sim\left|p^{(h, e)} / p^{(s)}\right| \ll 1 .
$$

Furthermore, the energy dominance [2] is disrupted for $\varepsilon^{(s)}$ and $p^{(s)}$, and their components.

\section{Conclusions}

The model of the material medium, for which the electrically neutral relativistically invariant state with non-zero energy density, pressure, concentrations of particles and antiparticles is possible, has been considered. At the same time particles and antiparticles are in the thermal equilibrium, but far from the chemical equilibrium state governed by the equality of their chemical potentials. In the considered case, the expression is true

$$
F^{(e)}-F^{(h)}=2 c\left(m^{2} c^{2}+p_{F}{ }^{2}\right)^{1 / 2} \approx 2 m c^{2}\left[1+(1 / 2)\left((4 / \pi-1) \alpha\left(k_{B} T_{\Lambda} / m c^{2}\right)^{2}\right)^{2 / 3}\right] .
$$

This non-equilibrium electrically neutral state exists due to the random electromagnetic field generated by spontaneous transitions between particles and antiparticles being in different quantum states. The average vector potential and intensity of this field are zero. But the average components of the energy-momentum density tensor in the random process of transitions between the states of particles and antiparticles are non-zero.

The energy density of the above vacuum-like state can be expressed in terms of its temperature $T_{\Lambda}$. The state electroneutrality requirement leads to the equality of Fermi momentum of particles and antiparticles, and the relativistic invariance requirement to the equation relating the Fermi momentum with the temperature.

If the energy density of the system considered is identified with the dark energy density, then temperature $T_{\Lambda}$ turns to be of the order of $10^{-5} \mathrm{~K}$ and Fermi velocity of particles and antiparticles $v_{\Lambda}=p_{\Lambda} / \mathrm{m}$ to be approximately $1 \mathrm{~cm} / \mathrm{s}$ ( $\mathrm{m}$ is the mass of electron). Furthermore, the dark energy density and cosmological constant are negative.

\section{References}

[1] Zel'dovich, Ya.B. (1968) The Cosmological Constant and the Theory of Elementary Particles. Soviet Physics Uspekhi, 11, 381-393. http://ufn.ru/ru/articles/1968/5/m/

[2] Zel'dovich, Ya.B. (1981) Vacuum Theory: A Possible Solution to the Singularity Problem of Cosmology. Soviet Physics Uspekhi, 24, 216-230. http://ufn.ru/ru/articles/1981/3/c/

[3] Gliner, E.B. (1966) Algebraic Properties of the Energy-Momentum Tensor and Vacuum-Like States of Matter. Soviet Physics JETP, 22, 378-382. http://www.jetp.ac.ru/cgi-bin/r/index/e/22/2/p378?a=list

[4] Chernin, A.D. (2008) Dark Energy and Universal Antigravitation. Physics-Uspekhi, 51, 253-282. http://ufn.ru/ru/articles/2008/3/cl

[5] Lukash, V.N. and Rubakov, V.A. (2008) Dark Energy: Myths and Reality. Physics-Uspekhi, 51, 283-289. http://ufn.ru/ru/articles/2008/3/d/

[6] Chernin, A.D. (2013) Dark Energy in Systems of Galaxies. JETP Letters, 98, 394-407. http://www.jetpletters.ac.ru/ps/2018/article_30426.pdf http://dx.doi.org/10.1134/S002136401319003X 
[7] Corda, C. (2009) Interferometric Detection of Gravitational Waves: The Definitive Test for General Relativity. International Journal of Modern Physics D, 18, 2275-2282. http://dx.doi.org/10.1142/S0218271809015904

[8] Abbott, B.P., et al. (2016) Observation of Gravitational Waves from a Binary Black Hole Merger. Physical Review Letters, 116, 061102-01-061102-16. https://journals.aps.org/prl/abstract/10.1103/PhysRevLett.116.061102 http://dx.doi.org/10.1103/physrevlett.116.061102

[9] de Groot, S.R., van Leeuwen, W.A. and van Weert, Ch.G. (1980) Relativistic Kinetic Theory. Principles and Applications. North-Holland Publishing Company, Amsterdam, New York and Oxford.

\section{Submit or recommend next manuscript to SCIRP and we will provide best service for you:}

Accepting pre-submission inquiries through Email, Facebook, Linkedin, Twitter, etc A wide selection of journals (inclusive of 9 subjects, more than 200 journals)

Providing a 24-hour high-quality service

User-friendly online submission system

Fair and swift peer-review system

Efficient typesetting and proofreading procedure

Display of the result of downloads and visits, as well as the number of cited articles

Maximum dissemination of your research work

Submit your manuscript at: http://papersubmission.scirp.org/ 